\title{
Gravity-Like Fields and Space Propulsion Concepts
}

\author{
Walter Dröscher ${ }^{1}$, Jochem Hauser ${ }^{2 *}$ \\ ${ }^{1}$ Institut für Grenzgebiete der Wissenschaft, 6010 Innsbruck, Austria \\ ${ }^{2}$ Faculty Karl-Scharfenberg, Univ. of Applied Sciences, Salzgitter Campus, 38229 Salzgitter, Germany
}

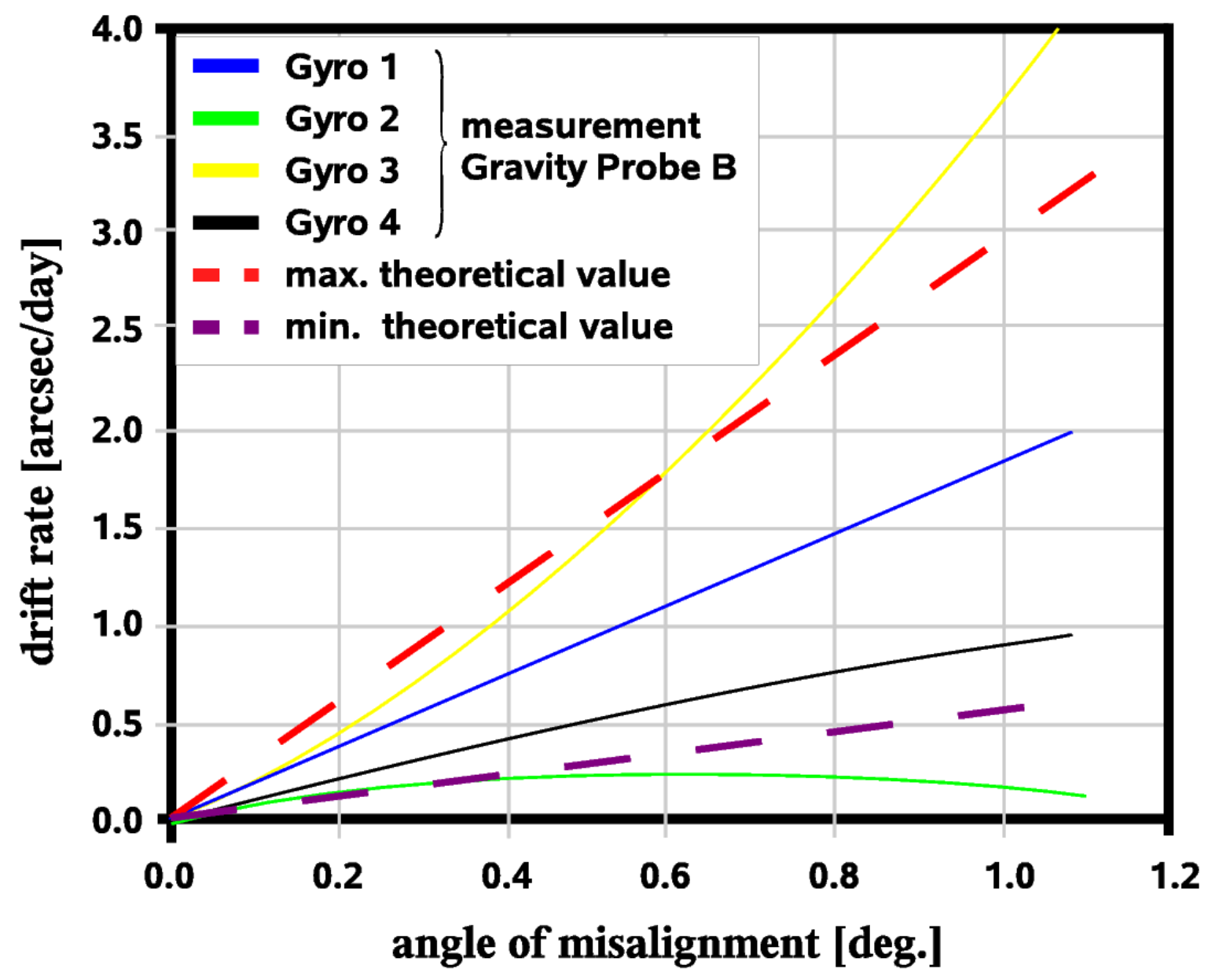

Figure 1. Experimental evidence for novel gravity-like fields. The figure shows an overlay of two pictures. The four solid curves, taken from the Stanford-NASA Gravity Probe B space flight experiment, ${ }^{1}$ show the directly measured misalignments of the four gyroscopes (gyro) employed in this experiment, made from niobium coated quartz spheres that are rotating at superconducting temperatures. The two dotted curves show the theoretical misalignment of the gyros calculated by EHT (Extended Heim Theory) caused by the presence of the postulated gravitomagnetic field, which, according to EHT, should result from the rotation of the cryogenic Nb coated quartz spheres (gyros). This gravitomagnetic field, being 18-20 orders of magnitude larger than classical relativistic frame dragging as predicted by $G R$ (General Relativity), is outside $G R$ and would denote a novel physical phenomenon. Moreover, the presence of this effect might cause gyro anomalies and render the data analysis much more challenging.

${ }^{* 1}$ Senior Scientist, Institut für Grenzgebiete der Wissenschaft, 6010 Innsbruck, Austria

*2 Prof., Faculty Karl-Scharfenberg, Univ. of Applied Sciences, 38229 Salzgitter, Senior member AIAA, member SSE.

Copyright $\odot 2008$ by Jochem Hauser. Published by the American Institute of Aeronautics and Astronautics, Inc. with permission.

$$
1 \text { of } 19
$$


In this paper we discuss the current state of the art on the existence of gravity-like fields, which are gravitational fields that cannot be described by conventional gravitation, i.e. by the accumulation of mass. The gravitomagnetic effect of these fields is 18-20 orders of magnitude larger than predicted by classical $G R$ frame dragging. The paper starts with an introduction to the present experimental basis for the existence of these novel gravity-like fields. The second section is dedicated to a discussion of the main physical features of a geometrized approach termed EHT (Extended Heim Theory), and to elucidate the underlying geometrization approach, extending Einstein's idea of the geometrization of physics by employing the additional concepts of Heim (1952, 1977) and Finzi (1955). As a consequence, $E H T$ predicts, as already published in 2002, the existence of two additional gravity-like fields that can be both attractive and repulsive that is, there exist six fundamental physical interactions, of which three are of gravitational nature. In section three, all experiments are discussed that have measured gravitomagnetic or gravity-like fields (acceleration), and EHT is utilized to calculate magnitude, direction, and physical features of these fields for all those experiments. Furthermore, a comparison with measured and $E H T$ results is performed. In particular, an analysis is presented for the recent set of experiments by Tajmar et al. (2007, 2008) utilizing a cryogenic $(\leq 30 \mathrm{~K})$ rotating Niobium ring as well as the recent experiments by Graham et al. (2007) employing a rotating superconducting lead disk. Emphasis is also given to the NASA-Stanford Gravity-Probe B gyro misalignment phenomenon (2007). GP-B was launched in 2004 to measuring the frame dragging effect of the Earth, as predicted by Lense and Thirring in 1918. During GP-B another anomaly was seen, namely a $10 \mathrm{~Hz}$ frequency shift indicating both deceleration and acceleration among the two gyro pairs. In Section four, the nature and type of the fundamental interaction(s), responsible for gravitomagnetic effects, is determined. Utilizing straightforward physical arguments it is demonstrated that none of the four known fundamental interactions can be the cause of these fields. In addition, in the meantime, the frame dragging effect was determined from the LAGEOS and LAGEOS II satellite data, and $G R$ was confirmed within 5 to $10 \%$ accuracy. Thus, the frame dragging effect is extremely small, and $G R$ cannot be responsible for any of the observed gravitomagnetic phenomena. This holds also true for the gyro misalignment and the frequency shift in the GP-B experiment. The GP-B team did a careful analysis of all physical effects that might have been responsible for the misalignment, e.g. electrostatic forces between the gyro housing and the gyro surfaces, but it seems that the phenomenon cannot be resolved completely. According to $E H T$, a substantial part of the observed gyro anomaly should be due to the action of the two additional predicted gravity-like fields. Therefore, there should still be room for the gyro misalignment effect of gravitomagnetic fields. In Section five, based on the results of $E H T$, a novel experiment for the generation of a vertical gravity-like field is presented that might serve as propulsion principle, depending, however, on the physical nature of gravity-like fields. In the Conclusions section the validity of the experiments is discussed along with the state of the physical model. The major consequences of gravity-like fields in the general area of technology (transportation), physics, and cosmology are outlined. Finally, recommendations are made to advancing the state of gravitomagnetic research, and to the possibility of developing fundamentally new technologies.

\section{Introduction to the Experimental and Theoretical Generation of Gravitomagnetic and Gravity-Like Fields}

Spaceflight, as we know it, is based on the century old rocket equation that is an embodiment of the conservation of linear momentum. Moreover, in our spacetime, special relativity puts an upper limit on the speed of any space-vehicle in the form of the velocity of light in vacuum. Thus, current physics poses severe constraints on space propulsion technology. These limitations can only be overcome if novel physical laws can be found. Therefore, during the last two decades, numerous experiments related to gravity shielding or gravitomagnetic interaction (coupling between electromagnetism and gravitation) were carried out, but eventually all proved to be incorrect.

In the 1990s a Russian scientist claimed to have measured gravitational shielding. A similar claim was made by an American scientist several years later. However, in their recent paper Woods et al. ${ }^{10}$ have delivered overwhelming experimental evidence that these two claims cannot be substantiated. This kind of gravitational shielding simply does not exist. In 1997, a Japanese free fall experiment using an encapsulated spinning gyro ${ }^{6}$ reported differences in free fall time depending on the direction of rotation. The authors concluded that an asymmetry (parity violation) caused the generation of anti-gravity. It is interesting to see that the following three sets of experiments described below, also observe an asymmetry between right and left rotations. However, the equations of motion for a free falling body in an atmosphere are highly complex, because of aerodynamic lift and drag (pressure drag, friction drag) that also may be time dependent. Although the spinning gyro was encapsulated, it might have transferred angular momentum, for instance, by friction effects, to the free falling body causing a rotation of the body. This rotation would have changed the free fall time. If the gyro had a small geometrical asymmetry, a rotation in one direction $(18,000 \mathrm{rpm})$ might cause a transition from laminar to turbulent flow, and thus may lead to a different transfer in angular momentum, depending on the direction of rotation. Since the authors do not address these possible sources of error, it cannot be decided 
whether or not this experiment is reliable, and therefore it was not considered in the current experimental analysis. This experiment was conducted at room temperature. It has not been investigated in how far such an effect is predicted by $E H T$.

However, on 23 March 2006, the European Space Agency (ESA), on their webpage, announced credible experimental results, reporting on the generation of gravitomagnetic (frame dragging field) and gravity-like fields (acceleration or artificial gravitational field, details see Sec. IIII in the laboratory. Since then, substantial progress has been made on the experimental front. Since 2006, Tajmar et al. have refined their experimental setup, repeating their experiments, and in July 2007, Graham et al. published a paper seeing a similar effect for a superconducting lead disk, but using a completely different measurement technique. In addition, in September 2007 Tajmar published a comparison between the two sets of experiments. Further results were recently published by Tajmar (2008) in, ${ }^{16}$ confirming earlier measurements. Moreover, in 2007 results of the Stanford-NASA Gravity Probe B (GP-B) $)^{1}$ experiment became available. GP-B employed four small, extremely precise gyroscopes as its main tool for detection of geodetic (spacetime deformation by a static mass) and frame dragging (spacetime twisting by a rotating mass) effects. Since in GP-B two pairs of counter-rotating superconducting niobium coated quartz spheres are utilized, the two gyros in each pair should feel the presence of the gravitomagnetic field as generated by the other. This situation is similar to Tajmar's and Graham's experiments, see Sec. A Thus, according to EHT, first, a gravitomagnetic field should be observed, leading to substantial gyro misalignment (i.e., spacetime twisting acting as a force that pushes on the gyro axis and forces it out of alignment as it circles around the Earth) and second, a tangential gravity-like field should be observed (acceleration field), generated by the rotation of a gyro in the spatially nonhomogenous gravitomagnetic field of its partner, leading to acceleration and deceleration of the two gyros. Therefore, the misalignment of the orientation of the axes of the GP-B gyroscopes over time should not be completely explainable by conventional assumptions like electrostatic forces between the housing of the gyro and the gyro surface etc. The same holds true for the second gyro anomaly, namely the measured gyro frequency shifts in GP-B, meaning that one of the gyros in each of the two gyro pairs was accelerated over the measuring period of 10 months, while the other one was decelerated. This can be understood by the fact that an observer fixed to the surface of one of the uniformly rotating gyros (each pair considered separately) represents a non-inertial frame, $F^{\prime}$. In this frame, the spatially non-homogenous gravitomagnetic field $\mathbf{B}_{g}$ of $F$, generated by the rotation of the second cryogenic gyro, is therefore perceived as a time varying gravitomagnetic field. Thus, a gravity-like (acceleration) field occurs in $F^{\prime}$, acting in the plane of rotation in tangential direction, and thus should either accelerate or decelerate the gyro, depending on its sense of rotation, clockwise (CW) or counter-clockwise (CCW), for further details see Sec. B.

The generation of the gravitomagnetic and/or gravity-like field(s) in all three sets of experiments, Tajmar, Graham and GP-B seems to follow the same mechanism. A gravitomagnetic field is observed for a rotating $\mathrm{Nb}$ or $\mathrm{Pb}$ ring, sphere, or disk at a specified cryogenic temperature (somewhat above the critical temperature, $T_{C}$, for superconducting), and is many orders of magnitude larger than predicted by $G R$. A gravity-like field (gravitational acceleration field) can be generated by producing a time-dependent gravitomagnetic field. The ensuing gravitational force observed is more in the range of the electromagnetic force. In other words, the coupling constant for the gravity-like field must be completely different from common gravitational coupling. Hence, we are in the fortunate situation to have data for the validation of physical models, in contrast to current theoretical physics, in that three different types of experiments exist, employing very different measurement techniques, performed both on earth and in space, but reporting the same physical phenomenon.

Regarding the reliability of the experimental results, it should be mentioned that M. Tajmar et al. at the ARC Seibersdorf, Austria (a certified ESA test center) had carried out these experiments over a period of about three years prior to publication. The authors report that a rotating $\mathrm{Nb}$ ring of some $15 \mathrm{~cm}$ diameter generated a gravitomagnetic field below some $30 \mathrm{~K}$. In addition, every time the cryogenic $\mathrm{Nb}$ ring was subjected to angular acceleration at around 4 $\mathrm{K}$, a gravity-like field was measured in the plane of the ring in circumferential direction. The induced acceleration field was opposite to the angular acceleration, following some kind of gravitational Lenz rule. In addition, an acceleration field was also observed when the $\mathrm{Nb}$ ring was rotating with constant angular velocity, but undergoing a phase change that is, from the normal to the (superconducting ?) state. This was achieved by reducing the temperature below $9.2 \mathrm{~K}$, the critical temperature for $\mathrm{Nb}$. Moreover, no acceleration was measured when the $\mathrm{Nb}$ ring was in normal conducting state.

$G R$ predicts that any rotating massive body (Earth) drags its local spacetime around, called the frame dragging effect, generating the so-called gravitomagnetic field. Since spacetime itself gets twisted, a gyro whose axis of rotation remains invariant, should exhibit a misalignment, since our local coordinate system is no longer inertial. This effect, predicted by Lense-Thirring 1918, however, is far too small to be seen in a laboratory on Earth. For this reason the GP-B experiment was launched in 2004 after more than 40 years of preparation (see Sec. [III). On the other hand, the 
values measured by Tajmar et al. were about 18 orders of magnitude higher than predicted by $G R$, and therefore are outside $G R$. They cannot be explained by the classical frame dragging effect of $G R$ and represent a new kind of physical phenomenon. In other words, the cryogenic $\mathrm{Nb}$ ring, with a mass of about 100 grams, caused approximately the same frame dragging effect as a white dwarf, see Eq2

When analyzing Tajmar's experiments using EHT, it became obvious that an experiment could be devised, demonstrating the generation of a gravity-like field in the vertical direction (along the axis of rotation), most likely capable of lifting a body from the surface of the Earth. Sine this effect only occurs at very low temperatures it is surmised that a phase change takes place leading to some kind of boson coupling between electrons that do not necessarily have to form Cooper pairs, but instead might form some kind of Bose-Einstein condensate, causing these novel physical interactions. Based on calculations of EHT, the technical requirements like magnetic induction field strength, current density, supply power etc. should easily be met with present technology. Required values for such an experiment are substantially lower than for the previously proposed experiments that assumed fermion coupling to achieve vacuum polarization. $^{22}$

Extreme caution is needed when announcing novel physical interactions. Several years ago, a novel physical interaction (fifth force) was announced by a group of physicists at Purdue University and Brookhaven National Laboratory (Fischbach, 1986) who claimed to have found experimental evidence for a distinct deviation from Newton's law at intermediate distances between $1 \mathrm{~m}$ and $1 \mathrm{~km}$. After four years of elaboration and 12 more experiments, agreement was reached that there was no fifth or sixth force. This time, the situation is different in that no geological structure or chemical composition of the environment is of importance. The physical effects measured by Tajmar and Graham are large, and should be clearly reproducible, independent on location and geological formation.

What does theoretical physics have to say about these experiments and physical phenomena? The answer is very short, namely there is no place for these experimental results. According to currently favored String theory or any other supersymmetry theory nothing should have been measured. What about alternative theories? At present, there are no theories capable of completely explaining these experiments. Using the geometrization approach of $E H T$ results in postulating two additional fundamental gravitational like interactions, which are subsequentely utilized to analyze all three types of experiments, see Sec. III] However, experiments are producing numerous new results, and no final conclusions from $E H T$ are available at present.

Regarding the construction of an advanced propulsion device, an additional base experiment might be feasible, in which the gravity-like field is directed along the axis of rotation, and thus could provide a more direct mechanism for a field propulsion principle working without propellant. Naturally, such a propellantless propulsion system would be far superior to any existing propulsion technology, while its technology might be substantially simpler than chemical, fission, and fusion rockets. There is, of course, insufficient knowledge at present whether such a device is technically feasible. On the other hand, benefits would be enormous, justifying substantially more extensive research in this field.

\section{Theoretical Model of Gravitomagnetic and Gravity-Like Fields}

In a series of articles since 2002 the existence of additional gravity-like fields was proposed by the authors. Here only a brief non-mathematical account of the fundamental assumptions of the underlying physical model termed $E H T$ (Extended Heim Theory) is presented. According to EHT, each point in 4-dimensional spacetime is equipped with an 8-dimensional internal symmetry space (tangent space), called Heim space $\mathrm{H}^{8}$. Heim space comprises four subspaces. Combining these subspaces by employing certain selection rules, a set of partial metric tensors is obtained, forming a polymetric that represents all of the known fundamental interaction forces. The partial metrics are interpreted as physical interaction field or group of elementary particles. As a consequence, this geometric approach predicts two further fundamental physical interaction fields, in addition to the four experimentally known ones. The nature of these two novel fields is gravity-like.

The six fundamental interactions emerge in our 4-dimensional spacetime and represent real physical fields carrying energy. The two additional interaction fields are identified as gravitophoton interaction (i.e., the conversion of photons into a gravity-like field), and quintessence or vacuum interaction (a conversion of photons into a repulsive type of gravity-like field). The gravitophoton interaction is mediated by two massless, so called gravitophoton particles, one which is gravitationally attractive, and the other one is gravitationally repulsive. The massless quintessence or vacuum interaction particle mediates a very weak repulsive gravity-like force that is much smaller in magnitude than the gravitophoton interaction.

The important question is under which experimental circumstances do these interactions manifest themselves as real physical phenomena? The answer is that in the experiments by Tajmar et al, Graham et al. as well as in the Stanford-NASA Gravity Probe B experiment these novel physical phenomena become apparent, which will be 


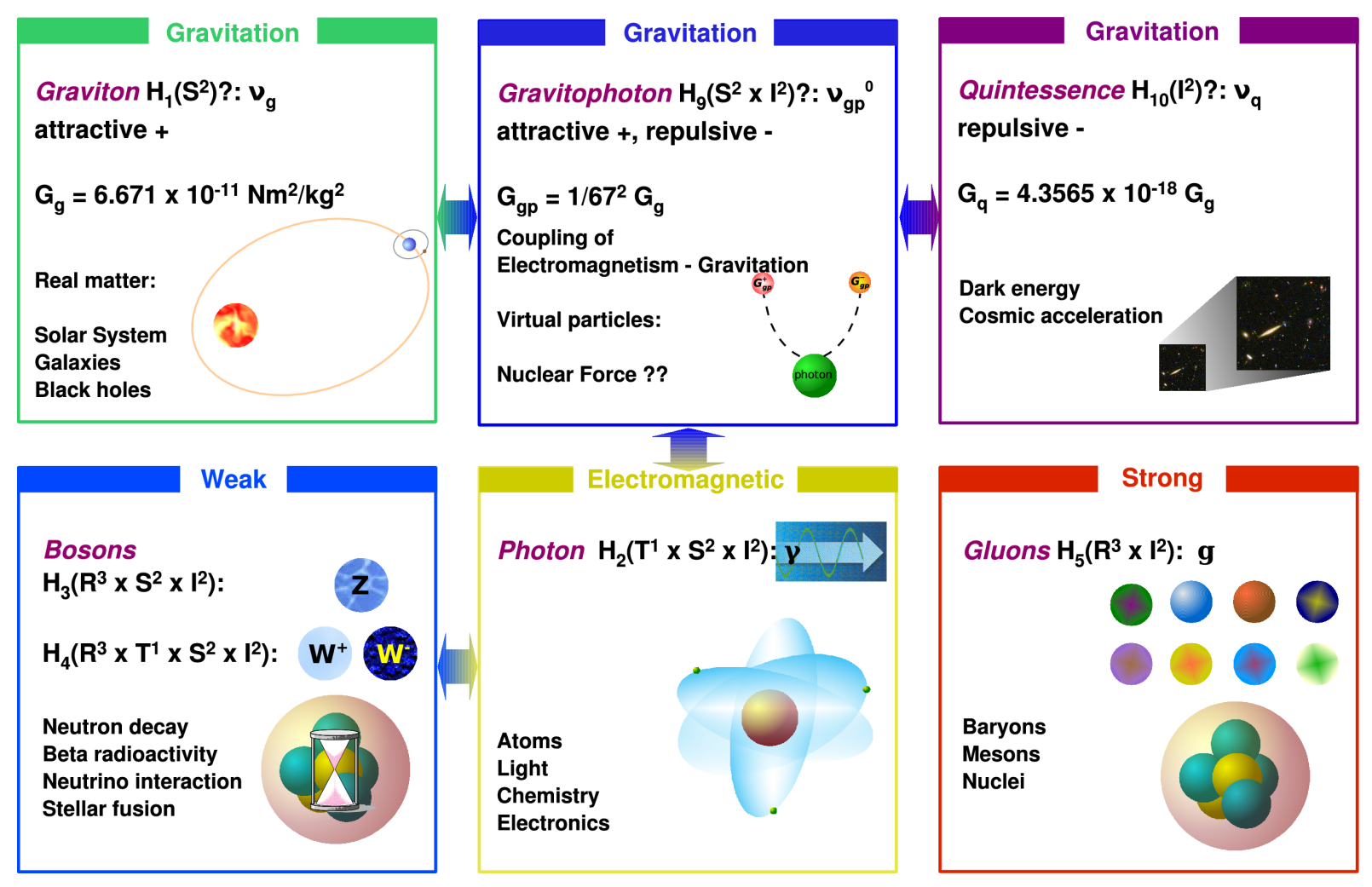

Figure 2. Six fundamental forces are predicted by EHT. Three of them are gravity-like fields, mediated by the graviton (attractive), gravitophotons (attractive and repulsive), and the quintessence particle (repulsive). The coupling strengths for the three gravitational fields were calculated from set theory, i.e., their values are obtained from pure mathematical considerations. $H_{\ell}$ indicates the type of Hermetry form, that is, the partial metric tensor, which corresponds to this physical quantity, see Tables 1 and 2 in. ${ }^{25,26}$ The arguments of a Hermetry form indicate the subspace coordinates that are forming this metric tensor. The internal symmetry space comprises four subspaces, namely $\mathbb{R}^{3}$ responsible for mass, $\mathrm{T}^{1}$ accounting for charge, $\mathrm{S}^{2}$, for organization, and $\mathrm{I}^{2}$ for information. Hermetry forms can be converted into different Hermetry forms by experimentally canceling certain subspace coordinates. This is how mixing of particles can be achieved.

discussed in some detail in the subsequent section.

Hence, according to $E H T$, the gravitational force that we experience is comprised of three different fundamental interactions, having six interaction quanta, namely two different neutral gravitophotons that can decay via two modes. The first mode gives a decay into a graviton (the usual graviton of quantized $G R$ theory) and the repulsive quintessence particle. The second mode results in two gravitophotons one being attractive, the other one repulsive. The gravitophoton and quintessence interactions should be invariant under an $\mathrm{SO}(4)$ group (not $\mathrm{SO}(3,1)$ ), see, ${ }^{26}$ Table 2. The laws of momentum and energy conservation are strictly obeyed, but both ordinary and non-ordinary matter must be accounted for. ${ }^{26}$

This means that the gravitational constant $\mathrm{G}$ contains contributions of all three gravitational constants, termed $G_{g}, G_{g p}$ and $G_{q}$, respectively. The quintessence interaction, however, is much smaller than the first two contributions. For further details see. ${ }^{22}$

The main idea of $E H T$ is that spacetime possesses an additional internal structure, described by an internal symmetry space $\mathrm{H}^{8}$, which is attached to each point of the spacetime manifold. The internal coordinates of $\mathrm{H}^{8}$ depend on the local (curvilinear) coordinates of spacetime. This is analogous to gauge theory in that a local or gauge transformation is used. In gauge theory it is the particles themselves that are given additional degrees of freedom, expressed by an internal space. Consequently in the geometrization of physics, it is spacetime instead of elementary particles that has to be provided with internal degrees of freedom. The introduction of an internal space has major physical consequences. The structure of $\mathrm{H}^{8}$ determines the number and type of physical interactions, and subsequently leads to a polymetric. This means that spacetime comprises both an external and internal structure. In general, only the external structure is observed, but it has long been known experimentally that matter can be generated out of the vacuum. This is a clear sign that spacetime has additional and surprising physical properties. Therefore, any physical theory that aims at describing physical reality, needs to account for this fact. Since $G R$ uses pure spacetime 
only, as a consequence, only part of the physical world is visible in the form of gravitation.

This idea was first conceived by the German physicist B. Heim. A similar principle was mentioned by the Italian mathematician B. Finzi. The polymetric tensor resulting from this concept is subdivided into a set of sub-tensors, and each element of this set is equivalent to a physical interaction or group of particles, and thus the complete geometrization of physics is achieved. This is, in a nutshell, the strategy chosen to accomplish Einstein's lifelong goal of geometrization of physics $a^{\text {a }}$

It must be noted that this approach is in stark contrast to elementary particle physics, in which particles possess an existence of their own and spacetime is just a background staffage. ${ }^{20}$ In $E H T$, considered as the natural extension of $G R$, matter simply is a consequence of the additional internal physical features of spacetime. These two physical pictures are mutually exclusive, and experiment will show which view ultimately reflects physical reality. It is, however, well understood that the concept of a pointlike elementary particle is highly useful as a working hypothesis in particle physics.

The approach of $E H T$ is substantially different from $G R$ and leads to the complete geometrization of physical interactions. But it reduces to $G R$, if the internal symmetry space is no longer considered.

Naturally, the number and type of interactions depend on the structure of internal space $\mathrm{H}^{8}$ whose subspace composition was determined in. $., 9,25,26$ Contrary to the ideas employed in String theory, see for example, ${ }^{21} \mathrm{H}^{8}$ is an internal space of 8 dimensions that, however, governs physical events in our spacetime.

The crucial point lies in the construction of the internal space whose subspace composition should come from basic physical assumptions, which must be generally acceptable. In other words, since $G R$ does not possess any internal structure, it has a very limited geometrical structure, namely that of pure spacetime only. Because of this limitation, $G R$ cannot describe other physical interactions than gravity, and consequently needs to be extended. $E H T$ in its present form without any quantization, i.e., not using a discrete spacetime, reduces to $G R$ when this internal space is omitted. The metric tensor, as used in $G R$, has purely geometrical character, which means it is of immaterial character only, and does not represent any physics. Consequently, the Einsteinian geometrization principle is equating the Einstein curvature tensor, constructed from the metric tensor, with the stress tensor, representing energy distribution. In this way, the metric tensor field has become a physical object whose behavior is governed by an action principle, like that of any other physical entities. In $E H T$ the internal space $\mathrm{H}^{8}$ is associated with physics through the introduction of three fundamental length scales, constructed from Planck quantities.

\section{Experimental Basis for Gravitomagnetic and Gravity-Like Fields}

All experiments performed so far contain a rotating mass that, when cooled to cryogenic temperatures (depending on the material), suddenly shows some kind of extreme frame dragging (spacetime twisting), which means a $\mathbf{B}_{g}$ field is generated whose magnitude is about 18-20 orders larger than classical frame dragging from $G R$.

Classical frame dragging only depends on mass distribution, i.e., on the moment of inertia, but does not exhibit any dependence on the type of material. This is obvious, since mass can be considered as gravitational charge. However, in contrast to classical frame dragging the observed frame dragging effect in the experiments by Tajmar et al. depends on the type of material and also on temperature. Moreover, in contrast to classical frame dragging, the cryogenic (Tajmar) frame dragging depends on the direction of rotation (pronounced asymmetry between clockwise and counterclockwise directions). This distinction between left and right is also termed parity violation.

\section{A. Gravitomagnetic and Gravity-Like Field Experiments}

The experimental situation distinguishes two types of phenomena, namely the generation of gravitomagnetic fields $\mathbf{B}_{g}$ (space time twisting), dimension 1/s, that are measured by gyrometers. In case the total time derivative of the $\mathbf{B}_{g}$ changes, a gravity-like field (gravitational acceleration) is observed, measured by accelerometers. Hence, the existence of a $\mathbf{B}_{g}$ field is a necessary but not sufficient condition for the existence of a gravity-like field. Furthermore, from the table of Hermetry forms, see Tables 1 and 2 in, ${ }^{26}$ it is deduced that two different types of gravitomagnetic $\mathbf{B}_{g}$ fields exist, which are represented by the neutral gravitophoton particle $v_{g p}^{0}$ that, depending on experimental conditions, can decay via two different modes, see ${ }^{25,26} \mathrm{~b}$ The first decay mode, which is believed to occur in the experiments by Tajmar et al., $v_{g p}^{0} \rightarrow v_{g}+v_{q}$, delivers a graviton and quintessence particle. The corresponding gravity-like field produces an acceleration in tangential direction in the symmetry plane of the accelerated rotating cryogenic ring and follows some kind of Lenz rule, i.e, it is directed against its origin, which is the mechanical acceleration of the ring. The

\footnotetext{
${ }^{\text {a }}$ There is of course a second aspect, namely the quantization of the spacetime field.

${ }^{\mathrm{b}}$ These two papers provide a more comprehensive and detailed analysis of both experiments and theory.
} 
second decay mode, $v_{g p}^{0} \rightarrow v_{g p}^{+}+v_{g p}^{-}$, gives a positive (attractive) and negative (repulsive) gravitophoton. This mechanism is deemed to be responsible for the generation of a vertical gravity-like field. In other words, gravitomagnetic fields are generated if the cryogenic $\mathrm{Nb}$ ring (or disk, sphere etc.) is rotating at constant angular speed. A tangential gravity-like field is generated if the cryogenic $\mathrm{Nb}$ ring (or disk, sphere etc.) is subject to angular acceleration. In any case, an effect is observed only when the temperature is below a critical cryogenic temperature, which seems to be somewhat higher than the superconducting temperature, $T_{C}$, of the ring material. Applying a mechanical acceleration, means that the partial time derivative $\partial \mathbf{B}_{g} / \partial t \neq 0$. According to $E H T$ a vertical gravity-like field should be produced if a current is induced into the superconducting uniformly rotating $\mathrm{Nb}$ ring, in place of the mechanical acceleration.

Analyzing the GP-B experiment, there should exist two anomalies regarding the two gyro pairs. First, the gravitomagnetic fields generated by the rotation of the four cryogenic $\mathrm{Nb}$ coated quartz spheres (gyros) should cause a misalignment of their gyro axes, see Fig 6 . Second, since the gravitomagnetic fields are spatially non-homogeneous that is, $\mathbf{B}_{g}=\mathbf{B}_{g}(\mathbf{r})$, an observer in the coordinate system of the rotating gyro one (regarding a single gyro pair), which has been fixed to its surface, will experience a time dependent gravitomagnetic field produced by the second gyro c) Therefore, gyro one should be subject to a tangential force in its plane of rotation. The same holds true for gyro two. Since the gravity-like force is acting in the same direction, one gyro should be accelerated, the other one decelerated, because they are counter-rotating. This situation will be discussed further in Sec. B

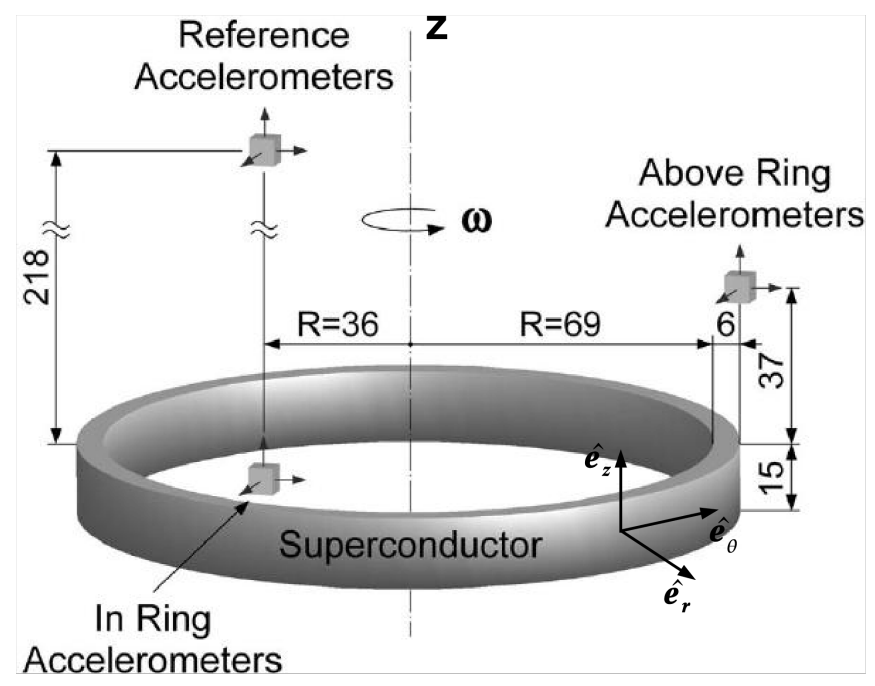

Figure 3. Rotating cryogenic ring (Niobium), modified from Tajmar et al., see ref. ${ }^{13}$ All dimensions are in mm. A cylindrical coordinate system $(r, \Theta, z)$ with origin at the center of the ring is used. Coordinate axes were placed on the surface of the ring for visualization purposes only. In-Ring accelerometers measured a gravitational acceleration of $-1.4 \times 10^{-5} \mathrm{~g}$ in the azimuthal (tangential, $\Theta$ ) direction when the ring was subjected to angular acceleration, see Fig. $8(\mathrm{a})$ ref. $^{13}$ for the so called curl configuration that comprises a set of four accelerometers. In an earlier publication, see Fig. 4a) in, ${ }^{12}$ an acceleration field of about $-10 \times 10^{-5} \mathrm{~g}$ was measured for a single accelerometer. The acceleration field did not depend on angular velocity $\omega$. The more recent experiment employed a set of 4 in-Ring accelerometers and confirmed the rotational character of this field. When the direction of rotation was reversed, the acceleration field changed sign, too.

In the experiments by Tajmar et al. no acceleration was measured (averaged) when the $\mathrm{Nb}$ ring was in the normal conducting state. Figure 4 was modified from Tajmar et al., see. ${ }^{13}$

The gravity-like field acts in circumferential direction, located in the plane of the ring, and is opposite to the angular acceleration of the ring. Thus, a propulsion force cannot be directly generated. In the right picture of Fig. 4 , the ring is in normal conducting state, and regardless of its state of mechanical motion, no gravity-like field is observed ${ }^{d}$. This is an indication that the presence of electron pairs (boson coupling) could be responsible for the generation of the acceleration field. From a theoretical point of view, Cooper pairs might not be necessary to generate a gravity-like field. Once the gravitomagnetic field exists, its temporal or spatial variation should give rise to a gravity-like field.

When analyzing the experiments by Tajmar et al., it occurred that an experiment could be devised, demonstrating the generation of a gravity-like in the vertical direction (along the axis of rotation). Due to the boson coupling (Cooper

${ }^{\mathrm{c}}$ There could be a self-induction effect, i.e., the gyro rotating in its own gravitomagnetic field causing a tangential force. However, the experiments by Tajmar et al. do not observe this type of phenomenon. Therefore, only the field of the second gyro is used.

${ }^{\mathrm{d}}$ The terms acceleration field and gravity-like field are used synonymously 


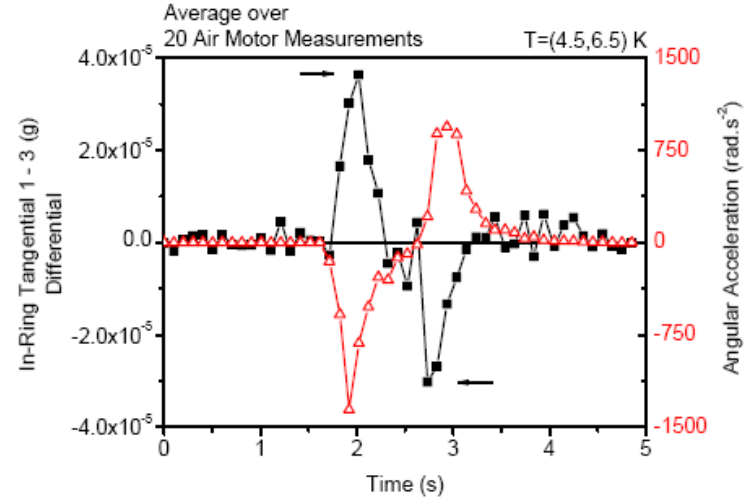

(a) Superconducting.

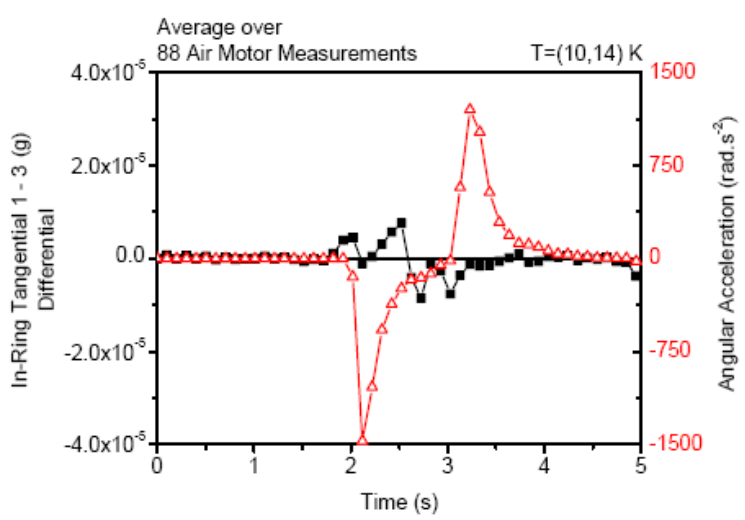

(b) Normal Conducting.

Figure 4. The Nb ring is in superconducting state (a). As soon as the ring is subjected to angular acceleration (red curve), a gravity-like field is produced, causing the accelerometers to measure an acceleration field, which is acting in the opposite direction (black curve). No signal is detected when the ring is in normal conducting state (b). Even if the ring is subjected to strong angular acceleration (red curve),the accelerometers do not see an acceleration field that is, the black curve oscillates around zero value. The difference to the left figure is clearly visible.

pairs or some kind of two-electron interaction) the technical requirements like magnetic induction field strength, current density, rotation speed of the cryogenic (superconducting) $\mathrm{Nb}$ ring, or current in the solenoid etc. should be straightforward to meet.

In 2004, the Gravity Probe-B experiment was launched, designed for a special purpose, namely to measure the Lense-Thirring effect. Since this effect is too small to be seen in a laboratory on Earth, a space flight experiment was set up. In other words, no frame dragging effects should be visible in any laboratory. In this experiment the Earth itself was used as a test body and measuring time was about 10 months, since the classical frame dragging effect accumulates over time.

The GP-B experiment used a set of four $\mathrm{Nb}$ coated quartz gyros, see above, to provide a coordinate system from the magnetic induction vector, generated in the superconducting state via the London effect. Since angular momentum of a gyro remains invariant, any torque on the gyro axis would be seen as a deviation (precession of the gyro axis). The gyros were coated with a thin sheet of niobium and were cooled below the critical temperature of $\mathrm{Nb}$. The magnetic induction field, generated by the London moment, served as coordinate axis, oriented toward a quasar. A substantial misalignment anomaly was indeed measured. This misalignment was seen while the gyros were rotating at constant angular frequency. The diameter of each sphere is $38 \mathrm{~mm}$. GP-B used two pairs of gyros, namely the pairs formed by gyros 1 and 2 as well as 3 and 4 . The gyros of a pair are rotating in opposite directions. Apart from the misalignment there exists another anomaly dealt with in Sec. B The two gyros within a pair had a separation distance of $75 \mathrm{~mm}$. The nature of the misalignment is still being discussed, but there seems to be room for the effect predicted by EHT and also measured by Tajmar et al. as well as Graham et al.

In the experiments by Tajmar et al. the cryogenic $\mathrm{Nb}$ ring is rotated together with is aluminum sample holder. The cross section area of the $\mathrm{Nb}$ ring is $A_{N b}=9.0 \times 10^{-5} \mathrm{~m}^{2}$, and for the sample holder $A_{A l}=7.8 \times 10^{-4} \mathrm{~m}^{2}$. The densities of $\mathrm{Nb}$ and $\mathrm{Al}$ are $\rho_{N b}=8.57 \times 10^{3} \mathrm{~kg} \mathrm{~m}^{-3}$ and $\rho_{A l}=2.70 \times 10^{3} \mathrm{~kg} \mathrm{~m}^{-3}$. According to Eq. 1 (derived from $E H T)$ the gravitomagnetic field of the cryogenic $\mathrm{Nb}$ ring with its aluminum sample holder is calculated as

$$
\mathbf{B}_{g}^{N b}=\frac{3}{5} 20 \pi \frac{1}{\sqrt{\alpha_{g p}}} \frac{G}{c^{2}} \frac{m_{e}}{m_{p}}\left(\rho_{N b} A_{N b}+\rho_{A l} A_{A l}\right) \omega=1.18 \times 10^{-5} \mathrm{rad} \mathrm{s}^{-1}
$$

where the factor $3 / 5$ accounts for the metric of the graviton (the contribution of the quintessence metric is 0 ), $G=6.67 \times 10^{-11} \mathrm{~m}^{3} /\left(\mathrm{kg} \mathrm{s}^{2}\right)$ is the gravitational constant, $\alpha_{g p}=1.31561 \times 10^{-42}$ is the coupling constant for the gravitophoton interaction, $c=2.99 \times 10^{8} \mathrm{~m} / \mathrm{s}$ the speed of light in vacuum, and $\mathrm{m}_{\mathrm{e}} / \mathrm{m}_{\mathrm{p}}=1 / 1836.15$ denotes the electron-proton mass ratio. An angular speed of $\omega=450 . \mathrm{rad} \mathrm{s}^{-1}$ was used.

Next, in order to compare this value with classical relativistic frame dragging, we calculate the gravitomagnetic field on the surface of a rotating white dwarf of about one solar mass, that is, $2 . \times 10^{30} \mathrm{~kg}, 6 . \times 10^{3} \mathrm{~km}$ radius, and a rotational velocity of $200 \mathrm{~km} / \mathrm{s}$. 


$$
\mathbf{B}_{g}^{W D}=\frac{2}{5} m_{W D} R_{W D}^{2} \frac{2 G}{c^{2}} \frac{1}{R_{W D}^{3}} \omega_{W D}=6.63 \times 10^{-6} \mathrm{rad} \mathrm{s}^{-1}
$$

which shows that the classical frame dragging as obtained from $G R$ through a relatively fast rotating and massive white dwarf is comparable to the gravitomagnetic field generated by the cryogenic $\mathrm{Nb}$ ring, having a mass of $100 \mathrm{~g}$ attached to its aluminum sample holder od several hundred gram. In other words, if the experiments by Tajmar et al. prove to be correct, this effect is outside $G R$ and denotes a novel physical phenomenon.

\section{B. Gravitomagnetic Experiments and Rotational Asymmetry}

In this section we briefly allude to additional experimental facts, namely the observed asymmetries (sometimes denoted

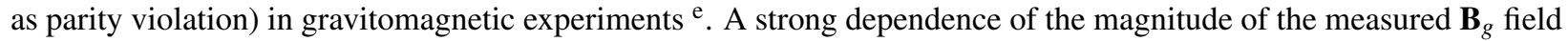
on the direction of rotation of the $\mathrm{Nb}$ ring was observed in all three experiments that is, this effect was reported in all experiments, i.e. by Tajmar et al., Graham et al., and also for GP-B. Tajmar et al. in one of their recent papers ${ }^{14}$ , clearly see these phenomena, independent of the material utilized. In contrast, the tangential acceleration field was invariant of the direction of rotation (no asymmetry), see Fig. 4. EHT predicts, however, that an axial acceleration field should exhibit an asymmetry.
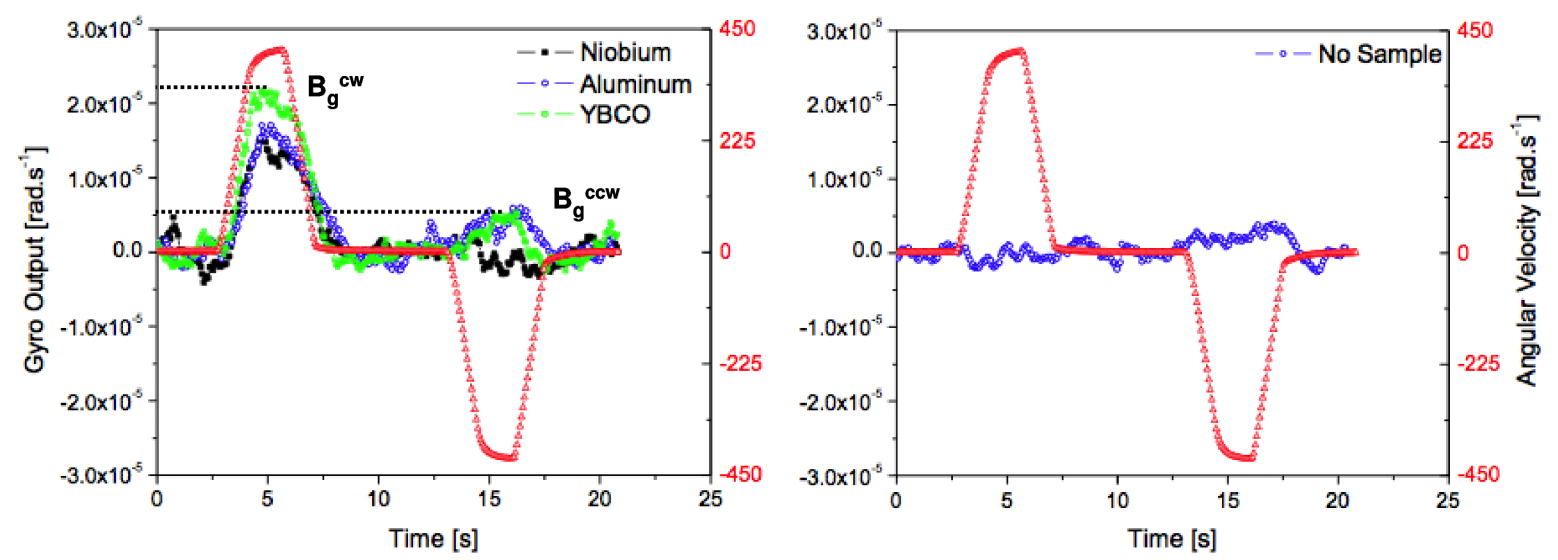

Figure 5. Measured asymmetry for the gravitomagnetic field $B_{g}$, (modified) from Tajmar et al., see ref. ${ }^{15}$ The red curve denotes the angular velocity of the ring. In these experiments the ring was not accelerated, but rotated at fixed speed. According to, ${ }^{26}$ the $B_{g}$ field is associated with Hermetry form $H_{9}$, which describes non-ordinary matter. The partial metric terms of this Hermetry form can be classified as symmetric or anti-symmetric. The ratio of maximal clockwise $(\mathrm{CW})$ and minimal counterclockwise $(\mathrm{CCW}) \mathrm{B}_{g}$ fields should be about 5 (average value), according to $E H T$, see text. The measured value is about 4.1. However, a tangential acceleration field should exhibit no asymmetry. On the other hand, an axial acceleration field, as predicted by EHT, should show a strong asymmetry factor of about 9.

Currently the combined set of Hermetry forms is only used as a classification scheme for particles and interactions as shown in recent papers ${ }^{25}$ and $^{26}$, where the existence of ordinary and non-ordinary matter is postulated. For instance, a neutral electron should exist, responsible for dark matter. It should be remembered that each individual Hermetry form (metric tensor that represents a physical interaction or particle group) comprises a set of partial metric terms, formed by their respective subspace coordinates. These partial terms can be classified as being symmetric or antisymmetric with respect to the interchange of their internal subspace coordinates. In general, any Hermetry form can be subdivided into symmetric and anti-symmetric partial terms. Each Hermetry form is defined by its corresponding potential

$$
g_{i k}\left(H_{\ell}\right)=\sum_{\mu, v \in H_{\ell}} g_{i k}^{(\mu v)}\left(H_{\ell}\right)=: \sum_{\mu, v \in H_{\ell}}(\mu v)
$$

which can also be written as

$$
g_{i k}\left(H_{\ell}\right)=\sum_{\mu, v \in H_{\ell}}(\mu v)_{S}+(\mu v)_{A}
$$

${ }^{\mathrm{e}}$ This asymmetry is an important physical effect and will be discussed in detail in a forthcoming paper. 
where indices $A, S$ denote the splitting of the partial terms in symmetric and anti-symmetric terms

$$
\begin{aligned}
&(\mu v)_{A}:=\frac{1}{2}[(\mu v)+(v \mu)] \\
&(\mu v)_{S}:=\frac{1}{2}[(\mu v)-(v \mu)] .
\end{aligned}
$$

From Eq. 5 one obtains directly

$$
(\mu v)_{A}=-(v \mu)_{A}, \quad(\mu v)_{S}=(v \mu)_{S}
$$

With each symmetric partial term $(\mu v)_{S}$ a fractional spin number $s_{n}= \pm 1 / n$ is associated, $n$ being the number of symmetric partial terms belonging to the same subspace (e.g. $\mathrm{S}^{2}$ ) in this Hermetry form. The number $s_{n}$ is positive for clockwise $(\mathrm{CW})$ and negative for counter-clockwise (CCW) rotation. This means that the spin number of a symmetric partial term does change sign when the direction of rotation is reversed. An anti-symmetric partial term $(\mu v)_{A}$ has fractional spin number $s_{n}=0$. Furthermore, the $\mathbf{B}_{g}$ field generated by a complete subspace metric that contains one or more anti-symmetric partial terms does not change sign if the direction of rotation is reversed. A subspace metric comprising symmetric terms only changes sign if the direction of rotation is changed.

Adding and subtracting the partial metric terms (physical potentials) with respect to their direction of rotation, therefore results in different gravitomagnetic fields $\mathbf{B}_{g}^{C W}$ and $\mathbf{B}_{g}^{C C W}$ for clockwise and anti-clockwise directions. Thus, according to $E H T$, the explanation for rotational dependence of the gravitomagnetic fields can be attributed to the number of symmetric and anti-symmetric partial metric terms in the Hermetry forms of the graviton and the quintessence particle, into which the neutral gravitophoton is decaying (experiments by Tajmar et al., Graham et. al., GP-B/f In these experiments, the gravitomagnetic fields are composed of the partial metrics of the graviton and quintessence particle, that is,

$$
\mathbf{B}_{g}^{C W}=\mathbf{B}_{g}\left(v_{g}\right)+\mathbf{B}_{g}\left(v_{q}\right)
$$

and

$$
\mathbf{B}_{g}^{C C W}=-\mathbf{B}_{g}\left(v_{g}\right)+\mathbf{B}_{g}\left(v_{q}\right) .
$$

As a result, a ratio of about 5 for $\mathbf{B}_{g}^{C C W}$ and $\mathbf{B}_{g}^{C W}$ fields should have been measured in the experiments by Tajmar et al. The fact that Graham et al. report the opposite behavior on the direction of rotation as seen by Tajmar et al. could mean that the $\mathbf{B}_{g}$ field lines are closed in the far field, and, since their measurements were taken much further away, outside the rotating disk, $\mathbf{B}_{g}$ field lines could have changed direction (i.e. sign change). g The fact that Tajmar has not seen a weakening of the gravitomagnetic field along the vertical axis could mean that two different types of gravitomagnetic fields exist.

The tangential acceleration field should not exhibit any asymmetry, since it is caused by gravitons, which only comprise symmetric partial terms. In the experiments by Tajmar et al., the acceleration of the $\mathrm{Nb}$ ring generated a time dependent $\mathbf{B}_{g}$ field, and the associated neutral gravitomagnetic particle decays via $v_{g p}^{0} \rightarrow v_{g}+v_{q}$. Only the action of the graviton can be observed, since the gravitational coupling constant of the quintessence particle is too small to have a local effect.

One should remember that there exist two decay modes for a neutral gravitophoton $v_{g p}^{0}$ particle, and the situation would be different in the generation of an axial acceleration field, which is produced by positive and negative gravitophotons via the second decay mode, $v_{g p}^{0} \rightarrow v_{g p}^{+}+v_{g p}^{-}$. In this case, a strong asymmetry factor of about 9 between the maximal and minimal acceleration fields should be observed. In addition, the axial acceleration field should be accompanied by a tangential acceleration field.

\section{Gravitomagnetic Fields from the Stanford-NASA Gravity Probe B Experiment}

The diameter of each quartz sphere is $38 \mathrm{~mm}$. GP-B uses two pairs of gyros one on top of the other, that is, their $\omega$ are on a straight line but antiparallel, namely the pairs formed by gyros 1 and 2 as well as 3 and 4 . The separation distance between two gyros (distance between the centers of the two spheres) is $75 \mathrm{~mm}$. The two dotted curves were determined theoretically, see Fig. 6 . They represent maximum and minimum drift values, due to spin-spin interaction between gyros used in the GP-B experiment. Theoretical values were obtained from EHT. According to EHT, a substantial part

\footnotetext{
${ }^{\mathrm{f}}$ We are well aware that this description deserves more detailed explanation.

${ }^{\mathrm{g}}$ Experiments by Graham et al. were performed in New Zealand. Being in the southern hemisphere should not cause a difference in the asymmetry
} 
of the observed gyro anomaly should be due to the two additional gravity-like fields, and should not be explainable by the four known physical interactions. h $^{2}$

In the following the fact will be used that the neutral gravitophoton can decay via graviton and quintessence particle or via positive (attractive) gravitophoton and negative (repulsive) gravitophoton (proposed experiment).
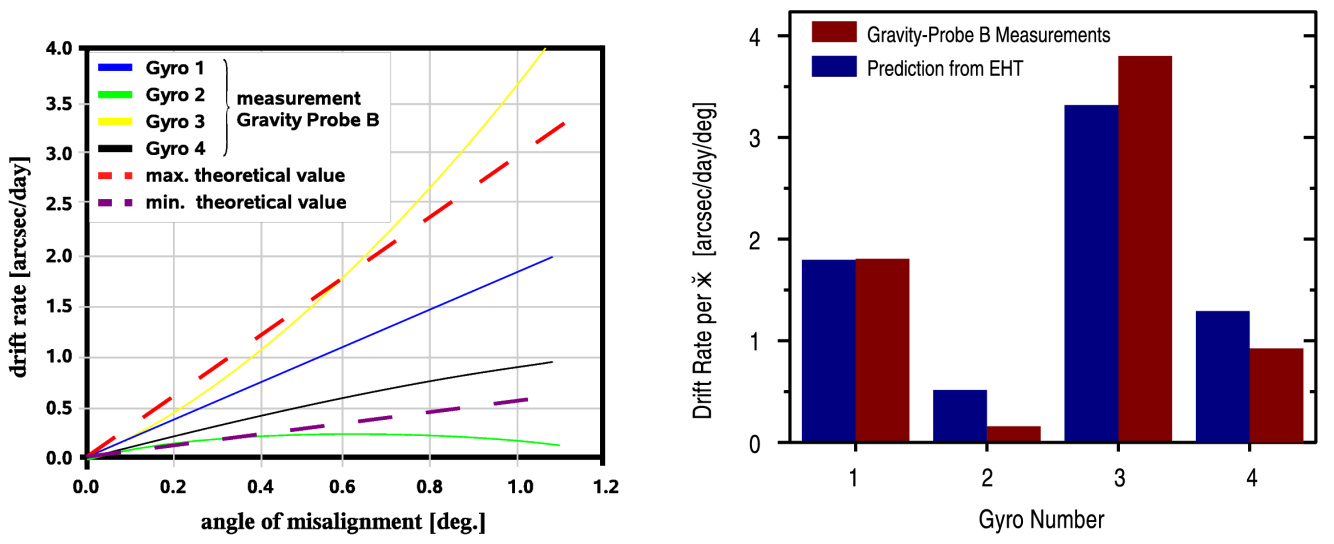

Figure 6. Comparison of measured misalignment values of Stanford-NASA Gravity Probe B experiment. Left picture shows an overlay of measured (solid lines) and maximum and minimum calculated drift rates as obtained from EHT. Right: Comparison of measured misalignment values for each of the four gyros of Stanford-NASA Gravity Probe B experiment with predictions from $E H T$.

The coupling to bosons is the prevailing mechanism in both experiments. In the experiments by Tajmar et al. the resulting gravitophoton decays into a graviton and a quintessence particle. For boson coupling experimental requirements, i.e., magnetic induction field strength, current densities, and number of turns of the solenoid are substantially lower than for fermion coupling (here the vacuum polarization is employed to change the coupling strength via production of virtual pairs of electrons and positrons) that was so far assumed in all our papers prior to 2006, see, for instance, refs. 222324

Regarding momentum conservation this is, obviously, not conserved if the action on the gravitational field itself is not taken into account. This means, regardless whether a gravitational field is generated by the mass of a planet or in the laboratory, it exerts a force on a material body. Since the beginning of space-flight, the gravitational fields of the planets have been used to accelerate a spacecraft. Any gravitational field modifies spacetime and the spacecraft simply follows a geodesic trajectory. In this sense, there is no medium needed for gravitomagnetic propulsion. The only difference to the well known gravity-assist technique is that instead of using gravitational fields of the planets, the spacecraft is moving by its proper gravitational field, generated by the conversion of photons into gravitophotons.

\section{Gravity-Like Fields from the Stanford-NASA Gravity Probe B Experiment}

The GP-B experiment uses two pairs of gyros, numbered 1-2 and 3-4. In each pair the upper gyro (number 1) rotates counter-clockwise, the lower one (number two) clockwise. Thus the resulting gravitomagnetic fields, denoted by $\mathbf{B}_{g}^{C W}$ and $\mathbf{B}_{g}^{C C W}$ have opposite directions and their magnitudes are related by $B_{g}^{C W}=5 B_{g}^{C C W}$, see Sec. B Apart from the misalignment of the gyro axes that was described in the previous section, it was also observed that the angular frequency of the gyros was changing during the experiment. At the start of the experiment, the average angular frequency was about $70.6 \mathrm{~Hz}$ for pair one, and $73.5 \mathrm{~Hz}$ for pair two. At the end of the experiment, after some 10 months, the frequency of the gyros was reported as 79.4, 61.8, 82.1, and $64.8 \mathrm{~Hz}$, respectively. Thus the observed frequency shift was almost the same in both pairs, namely $8.7 \mathrm{~Hz}$. Obviously, the set of all four gyros must satisfy the conservation of angular momentum. This means that the angular frequency of gyros 1 and 3 increased, while the frequency of gyros 2 and 4 decreased. So far, this effect has not been fully explained.

Although there exists a (slightly time dependent) coupling among all four gyros, for the sake of simplicity, the following analysis only considers the interaction between the two gyros within a pair. An average frequency value of $72 \mathrm{~Hz}$ is used. In the laboratory frame, $F$, the $\mathrm{CW}$ rotating gyro produces a $\mathbf{B}_{g}^{C W}$ field that is stationary, but not uniform. It varies both in direction and magnitude from place to place. Using the right-hand rule, the $\mathbf{B}_{g}$ line at the center of

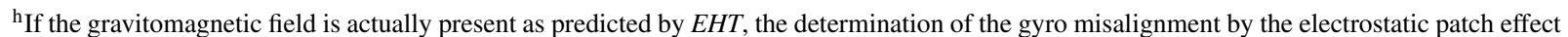
should not be completely able to account for the magnitude of the misalignment. Perhaps, GP-B might have seen a novel physical phenomenon, similar to Tajmar et al.
} 
the ring points in axial direction, along the negative $z$-axis 1 Now, for the gyro rotating in CCW direction, as seen from an observer in the frame $F$, the coordinate system fixed to the surface of this gyro, denoted by $F^{C C W}$, is seeing a time-dependent $\mathbf{B}_{g}^{C W}$ field, and consequently is subject to a tangential gravity-like force. Next, if the gravitomagnetic field generated by the $\mathrm{CCW}$ rotating gyro is regarded, a $\mathbf{B}_{g}^{C C W}$ field of different magnitude and opposite direction with respect to the $\mathbf{B}_{g}^{C W}$ field is produced. For an observer in the frame $F^{C W}$, the gravitomagnetic $\mathbf{B}_{g}^{C C W}$ becomes time dependent, and thus this gyro also experiences a tangential force. As the time dependent gravitomagnetic field is proportional to the rotation of the acceleration field, the acceleration acts in the same direction in both cases, since the two gravitomagnetic fields $\mathbf{B}_{g}^{C W}$ and $\mathbf{B}_{g}^{C C W}$ as well as the directions of rotation of the two gyros are of opposite sign. The time dependence of the gravitomagnetic fields is calculated from Eqs. 7 and 8 , respectively. It must, however, be considered that the acceleration needs to be symmetric, and thus the contribution of the quintessence metric is 0 . Thus, Eqs. 7 and 8 give the same acceleration. This means that a gravity-like field in circumferential (tangential) direction is generated, accelerating one of the gyros and decelerating the other one f $^{j}$ The gravitomagnetic field $\mathbf{B}_{g}$ responsible for the acceleration is given by

$$
\mathbf{B}_{g}=\frac{3}{5} 20 \pi \frac{1}{\sqrt{\alpha_{g p}}} \frac{G}{c^{2}} \frac{m_{e}}{m_{p}} \rho A \omega .
$$

Utilizing this value, the magnitude of the circumferential acceleration field in the symmetry plane of the gyro is calculated.

$$
g_{g p}=72^{3} \frac{v^{2}}{c} B_{g}
$$

where the factor $72^{3}$ is a theoretically calculated value. ${ }^{k}$ The tangential acceleration follows some kind of Lenz rule, i.e. acts against its cause, namely against the direction of the temporal change of $\omega_{N b}$. With measuring time $\mathrm{t}$ (approximately 10 months) the effect should accumulate, and eventually lead to a velocity change of

$$
\pm \Delta v=g_{g p} t=72^{3} \frac{v^{2}}{c} B_{g} t \approx 1.04 \mathrm{~m} / \mathrm{s} .
$$

The $\mathbf{B}_{g}$ field and $\Delta v$ can be obtained from experimental data. A simple estimate can be done as follows. Assuming a constant acceleration $g_{g p}$ in the circumferential direction during the 10 months period and using a radius of $r=$ $1.9 \times 10^{-2} \mathrm{~m}$ for the gyros (quartz spheres) as well as utilizing a change in frequency $\Delta v=8.7 \mathrm{~Hz}$, the average acceleration due to the gravity-like field is given by

$$
g_{g p}=2 \pi r \frac{\Delta v}{\Delta t}=6.28 \times 1.9 \times 10^{-2} \times \frac{8.7}{305 \times 86400} \approx 3.94 \times 10^{-8} \mathrm{~m} / \mathrm{s}^{2},
$$

which is a very small acceleration, but applied over a period of 10 months it lead to a velocity change of the gyros of $\pm 1.04 \mathrm{~m} / \mathrm{s}$. From this experimental value in combination with Eq. 10 the magnitude of the corresponding $\mathbf{B}_{g}$ can be determined. From Eq. 10 one obtains

$$
B_{g}^{G P-B}=1 / 72^{3} \frac{c}{v^{2}} g_{g p}=4.27 \times 10^{-7} 1 / \mathrm{s} .
$$

This value can then be compared with the theoretical $\mathbf{B}_{g}$ field of Eq. 9 where the spatial decline of this field needs to be taken into account. The second gyro is at a distance $d=7.5 \times 10^{-2} \mathrm{~m}$. Inserting the numbers from the experiment into Eq. 9 delivers the theoretical value for $\mathbf{B}_{g}$ at the center of the ring.

$$
B_{g}(0)=\frac{3}{5} 20 \pi \frac{1}{\sqrt{\alpha_{g p}}} \frac{G}{c^{2}} \frac{m_{e}}{m_{p}} \rho A \omega=3.77 \times 10^{-5} 1 / \mathrm{s}
$$

where $A$ is the cross section of the body, i.e. for the sphere $A=\pi \times r^{2}$. Assuming that the field at distance $d$ is that of a dipole

$$
B_{g}^{E H T}=\frac{r^{3}}{\left(r^{2}+d^{2}\right)^{3 / 2}} B_{g}(0)=5.39 \times 10^{-7} 1 / \mathrm{s} .
$$

${ }^{\mathrm{i}}$ The orientation of the laboratory frame $F$ is such that the positive z-axis is chosen to be parallel to the $\omega$-axis of the CCW rotating gyro

${ }^{\mathrm{j}}$ This is a preliminary qualitative analysis. A mathematically based analysis will also be performed.

${ }^{\mathrm{k}}$ The operator $\mathbf{v} \cdot \nabla$ has a factor $72^{3}$ assigned, the time operator $\frac{\partial}{\partial t}$ a value of $72^{1}$. 
Comparing the numerical results of the empirically determined gravitomagnetic field from Eq. 13 and the theoretical value determined from $E H T$, Eq. 15, there is an astonishingly good agreement. This could be taken as indication that such a gravitomagnetic field was actually present in the GP-B experiment. The EHT value should be higher, since it is valid for $T=0 \mathrm{~K}$. The result is even more astounding because in Eq. 14t the theoretically derived coupling constant for the gravitophoton interaction occurs, which was derived long before the GP-B results became known. The result is important also in another way, namely the density of the material seems to play a role. The superconducting $\mathrm{Nb}$ sheet obviously triggers the conversion of photons into gravitophotons that subsequently are decaying into gravitons and quintessence particles, but the quartz material seems to take part in the generation of the tangential gravitational field. Perhaps the process proceeds in two stages (the mechanism for the generation of gravitomagnetic and gravity-like fields is given $\mathrm{in}^{25,26}$ ). In stage one, a phase change needs to be produced in form of two-electron interaction causing an imaginary (virtual) current. In stage two, the imaginary charges (see Table 2 of non-ordinary matter $\mathrm{in}^{26}$ ) are converted into gravitophotons, because charge conservation has to be obeyed. This could have an influence on further experiments in that thinly coated configurations might have the same effect as, for instance, a full $\mathrm{Nb}$ ring. However, no data are available yet and theoretical predictions do not exist.

\section{Nature of Gravitomagnetic Phenomena and Physical Interactions}

From the experimental evidence presented above, it is assumed that gravitomagnetic measurements in the laboratory have demonstrated both the existence of a gravitomagnetic field and/or gravity-like field. The gravitomagnetic field is 18-20 orders of magnitude larger than predicted by $G R$. The question naturally arises how to explain these experimental findings. In order to account for gravitomagnetic phenomena by the four known physical interactions, it is necessary to identify those interaction(s) responsible for these effects. The subsequent analysis, based on qualitative physical arguments, leads to straightforward, but surprising conclusions.

- Gravity (Non-Newtonian) in the form of classical frame dragging as predicted by GR cannot be used to explain the existence of the gravity-like field or gravitomagnetic field. The Stanford-NASA GP-B experiment has shown the immense difficulties in measuring frame dragging for a body even of the mass of the Earth accumulated over a period of 10 months. Therefore, this effect cannot be measured for a small rotating ring or disk having a mass of about $100 \mathrm{~g}$ within a few seconds. Furthermore, the small mass of the ring in comparison to the mass of the Earth also rules out Newtonian gravity.

- Moreover, the Lense-Thirring frame dragging effect predicts the dragging of a moving object in the direction of rotation. The observed gravity-like field, however, acts opposite to the direction of rotation, following some kind of gravitational Lenz rule, and behaves more like an electromagnetic field. Thus common gravity cannot generate such a field.

- The physical explanation for these gravitomagnetic phenomena cannot be given by $G R$, even if the nonlinear equations were used, since no anomalous spacetime curvature was reported in the experiments.

- Conclusion 1: Gravitomagnetic phenomena are outside GR.

- All experiments by Tajmar et al. were electromagnetically very well shielded. Tajmar refined his shielding for about four years. ${ }^{15}$ The different measurement technique by Graham excludes any influence by electromagnetic fields.

- Conclusion 2: Electromagnetic interaction cannot take place.

- Both the weak and the strong force are of short range, i.e., on the scale of the nucleus. Gravitomagnetic and gravity-like fields, however, are of long range as is clearly demonstrated by the measuring process itself.

- In addition, the weak force cannot play a role, since there is no particle decay.

- Conclusion 3: Neither the weak nor the strong force can account for gravitomagnetic phenomena. No other physical interactions are known.

This leads to the following final conclusions, strictly based on experimental grounds.

1. Gravitomagnetic phenomena cannot be explained by any of the four known physical interactions. Therefore, the existence of at least one additional long range physical interaction is required. 
2. Moreover, in all experiments that produced a gravitomagnetic field, a strong asymmetry concerning the direction of rotation of the ring or disk (that is, clockwise or counter-clockwise) has been observed.

3. From the experiments it is clear, that these fields are gravity-like. This means that gravitational effects can be produced other than by the accumulation of large masses.

4. The existence of one or two additional physical interactions also demands the existence of one or two messenger particles (like the photon for electrodynamics), which must be bosons. These messenger particles are the carriers of the interactions. Since the fields observed are long range, the rest mass of these particles should be zero.

It is understood that extreme caution is needed when announcing novel physical interactions. The physical effects measured by Tajmar and Graham are large, and should be clearly reproducible independent on location and geological formation. Nevertheless, it is mandatory that other experimental groups reproduce these measurements, preferably using different measurement techniques.

For reasons based on the polymetric of $\mathrm{H}^{8}$, the existence of two additional physical interactions is assumed. Because of the asymmetry observed, it could be surmised that one of the interactions is attractive and the other one is repulsive. These additional gravitational interactions were termed gravitophoton (attractive and repulsive) and quintessence (repulsive) interactions. Their respective messenger particles are denoted as gravitophotons (attractive and repulsive) and quintessence (repulsive) particle.

Regarding the direction of gravity -like fields, the field of Tajmar et al. is in the circumferential direction, and needs a time varying neutral gravitophoton field. In the proposed vertical gravity-like experiment the time varying differential operator is replaced by the spatially varying operator (total derivative), which should lead to a completely different nature of the gravitomagnetic force that is much more amenable to space propulsion purposes, and should be directed along the axis of rotation.

In both cases the energy extracted from the vacuum is zero, since graviton and quintessence particles have negative and positive energy densities, respectively. With respect to the energy budget in the experiments by Tajmar et al., if only the energy of the gravitons can be measured, it should seem that energy conservation is violated. However, this would be a clear sign that the energy budget is not complete, because the positive energy density of the quintessence particle was not accounted for. In the vertical gravity-like field experiment, the total energy taken from the vacuum is also 0 . The two gravitophoton fields have opposite energy densities and add up to zero energy density. The gravitomagnetic force from positive gravitophotons is directed along the axis of rotation, while the gravitomagnetic force of the negative gravitophotons is in tangential direction.

\section{Novel Gravitomagnetic Experiments}

In the following two additional experiments are presented. First, a more accurate technique for the measurement of the acceleration field is suggested, and second, the experimental setup for the generation of a gravity-like vertical force (in the direction of the axis of rotation) is discussed. If such a field actually could be generated, it could be the basis for a gravity-controlled technology. A vertical gravity-like field could also serve as a demonstrator for a field propulsion principle without propellant, depending, however on the physical nature of the field. In such an experiment the cryogenic rotating ring, employed in the experiments by Tajmar et al., should be replaced by an insulating disk of a special material in combination with a set of superconducting coils. According to $E H T$, the physical mechanism is different from the experiments by Tajmar et al. in that the neutral gravitophoton decays into a positive (attractive) and negative (repulsive) gravitophoton, which should cause the gravity-like field to be directed along the axis of rotation of the disk.

\section{A. Acceleration Measurement for Gravity-Like Field: Photon Red- and Blueshifts}

In Tajmar's experimental setup a circular acceleration field of magnitude $a$ is generated by the nonuniform rotation of the (superconducting $N b$ ring) 1 . Here we suggest a modified setup to facilitate the measurement of the acceleration field, based on the gravitational mass, $m$, of the photon, which is equal in value to its inertial mass $h v / c^{2}$. Therefore, its energy $h v$ should be increased or decreased by $m a L$ when subjected to an acceleration of magnitude $a$ over distance $L$.

\footnotetext{
${ }^{1}$ According to theory, the superconducting state might not be needed. As soon as a $\mathbf{B}_{g}$ field is present and a time derivative is generated experimentally, an acceleration field should be observed. However, it could be that this field, because of its weakness, has escaped the measurements.
} 
In this novel experiment, in order to measuring the acceleration, $a$, accelerometers would be replaced by a circular fiber optics ring located in the plane of rotation, placed concentrically around the rotating ring. Since the acceleration field is equivalent to a gravitational field, a photon, similar to the experiment of Pound and Rebca in 1960, emitted inside the fiber optics ring, which is moving in a direction against the acceleration vector gains potential and loses kinetic energy, and thus is redshifted by

$$
v_{r}^{\prime}=v\left(1-\frac{a L}{c^{2}}\right)
$$

If the photon is emitted in the direction of the acceleration vector, it loses potential and thus gains kinetic energy and should be blueshifted by

$$
v_{b}^{\prime}=v\left(1+\frac{a L}{c^{2}}\right)
$$

where $a$ is the magnitude of the acceleration.

Since frequencies can be measured with much higher precision than mechanical acceleration, precision should be increased by several orders of magnitude. Moreover, this experimental set up would be much more robust against mechanical vibrations that plague accelerometer measurements. In particular, the accuracy should be sufficient to determine whether the strong asymmetry, between clockwise and counterclockwise rotation, as observed in the measurements for the $\mathbf{B}_{g}$ field, would also be present in accelerometer measurements (according to EHT, the tangential acceleration field should not exhibit any asymmetry, while the axial acceleration field (not yet measured but predicted), should show an asymmetry. Comparing the frequency shift of Eq. 16 with the relatively large red shift of a white dwarf $G M_{s} / R_{s}=5.3 \times 10^{12} \mathrm{~m}^{2} / \mathrm{s}^{2}$ it is obvious that the value $a L$ will be much smaller and more in line with the magnitude of the photon falling a distance $h$ in the gravitational field of the Earth, as measured by Pound and Rebca that is, $a L \approx g h$. This gravitational shift is much smaller than the natural line width, but employing the recoilless technique by Mössbauer (iron ${ }^{57} \mathrm{Fe}^{*}$ resonance, because of the metastable state the natural linewidth $\Gamma \approx 10^{-8} \mathrm{eV}$ is small for the $14.4 \mathrm{keV}$ photon (in reality there are six photon transitions)), Pound and Rebca were able to measure the energy change of the photon caused by its interaction with the gravitational field of the Earth. Therefore a similar technique should be applicable to the proposed experiment.

\section{B. Experiment for Vertical Gravity-Like Force}

This experiment (see Sec. III) should result in a measurable force acting in the direction of the axis of rotation that is, a vertical gravity-like field is generated. According to theoretical considerations this force should be generated by replacing the operator $\partial / \partial t$, that is the explicit temporal variation of the gravitomagnetic field $\mathbf{B}_{g} \mathrm{~m}^{\mathrm{m}}$ is replaced by the expression $(\mathbf{v} \cdot \nabla)$. With regard to the equivalence of 4-dimensional spacetime coordinates, both the convective and the time explicit operators applied to $\mathbf{B}_{g}$ should result in an acceleration field. However, the resulting acceleration fields should have different directions.

To minimize the modification of the current experimental setup of Tajmar et al., however, the following configuration is suggested: the sample holder is made of aluminum $(\mathrm{Al})$ and the rotating ring is made of niobium $(\mathrm{Nb})$. If the sample holder is made of teflon $(\mathrm{F})$, the gravitomagnetic field should be stronger. A solenoid (for instance copper $(\mathrm{Cu})$ ) is placed directly beneath this configuration, so that $\mathrm{Nb}$ ring and solenoid can act as transformer, the $\mathrm{Nb}$ ring counting as one turn only. A current of approximately $0.38 \mathrm{~A}$ is induced in the superconducting $\mathrm{Nb}$ ring by the $\mathrm{Cu}$ solenoid. The somewhat simplified formula below calculates the vertical acceleration field along the axis of rotation (averaged value, maximal and minimal values depending on the direction of current in the solenoid) that should be measured carrying out the experiment:

$$
g_{g p} \hat{\mathbf{e}}_{\mathbf{z}}=k k_{N b} k_{A l} \frac{1}{\pi} \frac{m_{e}}{m_{p}} \frac{v_{s h}^{2}}{c} \exp \left(-\left(\gamma-\frac{1}{\gamma}\right)^{2}\right) \omega_{I} .
$$

where the exponential factor accounts for some kind of resonance phenomenon and variable $\gamma=\left(\frac{I}{I_{0}}\right)^{2}$. $I$ denotes the induced current in the $\mathrm{Nb}$ ring, and the reference current is $I_{0}=0.38 \mathrm{~A}$ for the current experiment. In general it is a function $I_{0}=I_{0}\left(\omega_{I}\right)$. In the following it is assumed that $\gamma=1$.

A current is induced in the (superconducting) $\mathrm{Nb}$ ring by ramping up the current in the solenoid beneath this ring from zero to its final value, causing a time dependent magnetic flux. Quantity $\omega_{I}$ is the quantum mechanical angular frequency of the imaginary electron pairs in the $\mathrm{Nb}$ ring, $\sec ^{26} \mathrm{n}$, which should be formed when the phase transition

\footnotetext{
${ }^{\mathrm{m}}$ As produced by angular acceleration of the rotating ring in the Tajmar et al. experiments.

${ }^{\mathrm{n}}$ In this reference it is shown that in accordance with the recent work of G. Lisi ordinary matter is described by group $E_{8}$, but there should be a second group $E_{8}$ describing non-ordinary matter, e.g. gravitophotons, imaginary leptons, neutral electron etc.
} 
kicks in. It was determined from the quantum mechanically computed Fermi energy of the boson like electron pairs. From this energy the circumferential velocity of the imaginary electron pairs in the $\mathrm{Nb}$ ring was calculated as $2.16 \times 10^{5}$ $\mathrm{m} / \mathrm{s}$, and utilizing the value of the radius of the $\mathrm{Nb}$ ring, $7.5 \times 10^{-2} \mathrm{~m}$, their angular frequency $\omega_{I}$ was determined to $2.88 \times 10^{6} \mathrm{~Hz}$.

The temperature at which this transition occurs was determined for the $\mathrm{Al}$ sample holder (calculation of the fundamental coupling constants necessary) to about $15.9 \mathrm{~K}$. Most likely, a temperature below $9.2 \mathrm{~K}$ is needed for the cryogenic $\mathrm{Nb}$ ring. The coupling factors in Eq. 18 have the following values: $k=3, k_{N b}=[(1-\delta)(1-\delta a)]^{-1}-1$ with $\delta=1 / 24, a=1 / 8$, and $k_{A l}=1$. Quantities $m_{e}$ and $m_{p}$ denote electron and proton masses. The velocity $v_{s h}$ is the circumferential velocity of the sample holder, and the value of $v_{s h}=50 \mathrm{~m} / \mathrm{s}$ was used that was reached in the experiments by Tajmar et al. The acceleration field, $\mathbf{g}_{g p}$ is termed gravitophoton acceleration field, because it owes its existence to the conversion of photons into gravitophotons, see Tables 1 and $2 \mathrm{in}^{26}$, i.e., this conversion process seems to be the only path for ordinary matter to interact with non-ordinary matter.

In the experiments by Tajmar et al. a sensor is placed at a short distance $d$ above the $\mathrm{Nb}$ ring to measure the magnetic induction field, caused by the induced current in the superconducting rotating $\mathrm{Nb}$ ring. For $d=7.5 \times 10^{-2}$ $\mathrm{m}$, the magnitude of the magnetic induction field due to the London effect at the location of the field sensor is

$$
B=\frac{2 m_{e}}{e} \frac{1}{36} \omega_{I}=1.0 \times 10^{-6} \mathrm{~T}
$$

where the $B$ field is measured in Tesla. This means, a sensor reading of this value is equivalent to a current of $0.38 \mathrm{~A}$ in the $\mathrm{Nb}$ ring. Inserting the respective values into Eq. 18, one obtains for the magnitude of the acceleration field

$$
g_{g p}=3 \times \frac{1}{20.49} \times 1 \times \frac{1}{\pi} \times \frac{1}{1836} \times \frac{2.5 \times 10^{3}}{3 \times 10^{8}} \times 2.88 \times 10^{6} \times \frac{1}{9.81} g=6.2 \times 10^{-5} \mathrm{~g}
$$

where we inserted the quantum mechanical velocity of the electron pairs (Cooper pairs) ${ }^{0}$. It is suggested that, starting from the value $B \approx 1.0 \times 10^{-6} \mathrm{~T}$, the current in the $\mathrm{Cu}$ solenoid - which easily can produce this $\mathbf{B}$ field in the $\mathrm{Nb}$ ring should be varied in small steps, in order to measure the predicted value of the $g_{g p}$ acceleration field, since $\omega_{I}$ depends on the temperature of the $\mathrm{Nb}$ ring, and thus also the reference current $I_{0}$. Furthermore, Eq. 18 holds for $T=0 \mathrm{~K}$, and thus the specified values cannot be considered exact, but instead are approximate ones.

\section{Conclusions and Future Activities}

Since 2002 ideas for a geometric approach of describing physical interactions, termed EHT (Extended Heim Theory) were published. This approach predicts two additional physical interactions in the form of gravity-like fields, see for instance 792225 and ${ }^{26}$. A popular description of this research may be found in ${ }^{30} 313233$.

Numerous experiments by Tajmar et al. were carried out since 2003, and first published in 2006 report on the generation of gravitomagnetic (spacetime twisting) and gravity-like fields (acceleration) in the laboratory. The gravitational effects measured were about 18-20 orders of magnitude larger than predicted by the Lense-Thirring effect of GR. In other words, the rotating niobium ring, having a mass of some 100 grams as utilized by Tajmar et al., produces a gravitational effect similar to the mass of a a white dwarf. A popular description of their research may be found in. ${ }^{27,28}$ These experiments were repeated by Graham et al. in 2007, ${ }^{17}$ and most recently Tajmar et al. ${ }^{14}$ provided a comparison between the two experiments. Provided that the experiments of Tajmar and Graham are correct, a similar effect should have been observed in the Stanford-NASA Gravity Probe B experiment. The theoretical analysis of the GP-B anomalies based on EHT corroborates this idea.

In Section II, the geometrization concept of EHT was shown, which is based on the existence of an eightdimensional internal symmetry space, attached to each point of the conventional four-dimensional spacetime. This concept leads to exactly six fundamental physical interactions, three of them gravity like, but gravity can now be both attractive and repulsive.

In Section III, the experiments are discussed in detail and from the theoretical side, $E H T$, was used to predict the magnitude of the gyro misalignment by spin-spin interaction, caused by the generation of gravity-like fields acting among the two gyros in a gyro pair as well as the acceleration and deceleration observed in the two gyro pairs over the 10 months measuring period. The GB-P experiment utilized two pairs with counter-rotating gyros that exhibited an asymmetric misalignment behavior with regard to the direction of rotation. Theoretical predictions and measured misalignment were compared and gave reasonable agreement. It remains to be seen whether the electrostatic patch effect used to predict gyro misalignment by the Stanford team is capable to account for the magnitude of this effect and

\footnotetext{
${ }^{\mathrm{o}}$ It seems that for this experiment a superconducting state is needed, since a supercurrent has to be induced into the $\mathrm{Nb}$ ring.
} 
the observed accel-decel anomalies of gyro pairs in the post-flight analysis. Since the analysis of the misalignment is still ongoing by the GP-B team ${ }^{29}$ part of the misalignment of the two gyro pairs in the experiment might be attributable to the existence of the gravity-like field. In other words, the anomaly observed seems not to be completely explainable by classical effects, i.e., electrostatic forces etc. There seems to be room for the existence of gravity-like fields in the GP-B experiment. Therefore, the role of the electrostatic patch model used to explain a large part of the anomaly needs to be analyzed further, in order to revealing the true cause of the significant misalignment of the $\mathrm{Nb}$ coated quartz spheres in the GP-B experiment. The Lense-Thirring effect was already determined in late 2005 from LAGEOS and LAGEOS II satellites whose data were evaluated for a period of about 11 years, see the comprehensive paper by Ciufolini et al. ${ }^{19}$ Despite the smallness of the effect the analysis of the satellite data provided an accuracy between 5 to $10 \%$. Therefore, the Lense-Thirring (frame dragging) effect exists exactly as predicted by GR. Hence, there is no room using a modification of the Lense-Thirring effect as an explanation for the observed gravity-like fields. The explanation must be sought outside GR and requires novel physics. The acceleration phenomenon was discussed in detail and theoretical and experimental results are in good agreement. It could well be that the scientific value of the GP-B experiment might be in providing an independent proof of the existence of gravity-like fields.

In Section IV, EHT is used to perform an analysis of the experiments by Tajmar et al., Graham. et al., and GP-B in order to answer the salient question which of the four known fundamental physical interactions could be responsible for these enormous frame dragging like effects. Using straightforward physical arguments, it becomes evident that none of the four fundamental interactions can have caused these effects. Therefore the measurements, if correct, constitute a novel physical phenomenon, and thus additional physical interactions should exist. It is interesting to note, that none of the established physical theories (String theory, supersymmetry etc.) have predicted any effect like this, and thus have, at least, considered to be incomplete.

In Section V novel experiments are discussed that might lead to vertical gravity-like fields.

In summary, the present situation is characterized by the fact that a large number of experiments were performed over a period of more than four years, employing very different measurement techniques, but showing highly similar, but totally unexpected results. Measurement techniques in all experiments are clearly state of the art, in particular for the GP-B experiment and the latest experiments by Tajmar et al. ${ }^{16} \mathrm{p}$. Hence, the conclusion is that substantial experimental evidence for the existence of gravity-like field is available. It remains to be seen, whether in the case of the Gp-B experiment the misalignment of the orientation of the gyro axes and the change in the gyro rotation frequencies can be convincingly explained by classical physics. According to EHT, the misalignment of the orientation of the gyro axes has be attributed to a gravitomagnetic field, and the change in the gyro rotation frequencies needs to be traced back to a gravity-like field. If this were true, a rather solid experimental basis of three highly different experiments seeing the same novel physical phenomenon would exist. This would serve as a comprehensive experimental data set against which theoretical concepts have to be compared. In this regard the GP-B experiment might deliver highly interesting novel physical results. The current theoretical findings from EHT and their comparisons to experimental results are encouraging, though lots of open questions remain.

For the sake of clarity, the current situation (June 2008) is rephrased as follows. There is experimental data from three completely unrelated sources, employing completely different measuring techniques that have seen physical effects that are approximately 18-20 orders of magnitude larger than predicted by $G R$. In all experiments a phase transition occurred at low temperatures (not necessarily at $T_{C}$, the critical temperature for superconducting, but possibly two-electron interaction took place?). $G R$ cannot be used to explain these phenomena, even if the full nonlinear Einstein field equations were used, which should make no difference. The Lageos and GP-B experiments have clearly demonstrated that the inertial frame-dragging effect, even from celestial bodies, is extremely small and within $G R$. These facts provide evidence for novel physics in the form of additional fundamental forces.

How to proceed? It seems that there is sufficient reliable experimental data to start a comprehensive theoretical and experimental research program in gravity-like fields. There exists a physical model that can partly reproduce experimental facts, and also provide guidelines for an experiment generating a gravity-like field acting along the axis of rotation, while the ring or disk is rotating at constant speed. If such an experiment were successful, the goal of gravity-controlled technology (energy generation) or propulsion without propellant would be much closer. Naturally, a propellantless propulsion system would be far superior to any existing propulsion technology, while its technology might be substantially simpler than present technology of linear momentum conservation employed in chemical, fission or fusion rockets.

Moreover, gravity-like fields most likely would lead to novel technologies in the general field of transportation,

PThere was no time to use EHT to investigate the observed influence of the liquid helium circulation as reported in this experiment (June 2008). However, since Tajmar et al. have repeated their experiments several hundreds of times or even more, it seems reasonable to assume that previous measurements are still valid. 
and thus should be of major interest to the public and, in particular, to industry.

There is, of course, despite the rather solid experimental basis, insufficient knowledge at present whether these gravity-like fields are technically feasible and how they scale. The next step should therefore be to conduct a comprehensive and detailed study for determining the technical details of how to realize the proposed experiment for a vertical gravity-like field and to clarify the theoretical background. At the same time current experiments should be further refined and re-evaluated.

Needless to say, control of gravity would lead to completely new technologies, comparable to the advent of electricity and magnetism in the 19 th century.

\section{Acknowledgment}

The paper is dedicated to the eminent Dr. William Berry (ret.), head of Propulsion and Aerothermodynamics Division, ESTEC, ESA with whom the second author had the privilege to co-operate for several years.

The assistance by M.Sc. O. Rybatzki, Faculty Karl-Scharfenberg, Univ. Applies Sciences, Salzgitter Campus in preparing the figures is gratefully acknowledged.

The authors are particularly grateful to Dr. M. Tajmar, ARC Seibersdorf, Austria for providing general information, measured data as well as for numerous discussions and hints that helped in the comparisons between $E H T$ results and gravity-like experiments.

The authors are grateful to an anonymous reader for suggesting to utilize a white dwarf instead of a neutron star, in order to obtain a more meaningful comparison between the gravitomagnetic fields of the cryogenic $\mathrm{Nb}$ ring and an astronomical object.

The authors are most grateful to Prof. P. Dr. Dr. A. Resch, director of the Institut für Grenzgebiete der Wissenschaft (IGW), Innsbruck, Austria for his support in writing this paper.

The authors are indebted to Dr. A. Müller, Eichenau, Germany for reading the manuscript and his comments on the content of this article.

The second author was partly funded by Arbeitsgruppe Innovative Projekte (AGIP) and by Efre (EU) at the Ministry of Science and Education, Hannover, Germany.

\section{References}

${ }^{1}$ Stanford University: Gravity Probe B in a Nutshell, einstein.stanford.edu, August 2007.

${ }^{2}$ Einstein, A.: On the Generalized Theory of Gravitation, Scientific American, April 1950, Vol 182, NO.4.

${ }^{3}$ Heim, B.: Vorschlag eines Weges einer einheitlichen Beschreibung der Elementarteilchen, Zeitschrift für Naturforschung, 32a, 1977, pp. 233-243.

${ }^{4}$ Heim. B., Dröscher, W.: Strukturen der Physikalischen Welt und ihrer nichtmateriellen Seite, Resch Verlag, Innsbruck, Austria, 1996, 2nd ed. 2007.

${ }^{5}$ Cardone, F. and R. Mignani: Energy and Geometry, World Scientific, 2004.

${ }^{6}$ Haysaka, H. et al.: Possibility for the existence of anti-gravity: evidence from a free-fall experiment using a spinning gyro, Speculations in Science and Technology 20, 173-181.

${ }^{7}$ Dröscher,W., J. Hauser: Spacetime Physics and Advanced Propulsion Concepts, AIAA 2006-4608, 42nd AIAA/ASME/SAE/ASE, Joint Propulsion Conference \& Exhibit, Sacramento, CA, 9-12 July, 2006, 20 pp., (available as revised extended version 20 August 2006 at www.hpcc-space.de).

${ }^{8}$ Dröscher,W., J. Hauser: Current Research in Gravito-Magnetic Space Propulsion, Paper O-42, 7th International Symposium on Launcher Technologies, 2-5 April 2007, Barcelona, Spain, 16 pp.

${ }^{9}$ Dröscher,W., J. Hauser: Advanced Propulsion Systems from Artificial Gravitational Fields, AIAA 2007-5595, 43th AIAA/ASME/SAE/ASE, Joint Propulsion Conference \& Exhibit, Cincinnatti, OH, 8-11 July, 2007, 15 pp.

${ }^{10}$ Woods C. et al.: Gravity Modification by High Temperature Superconductors, AIAA 2001-3363, 37 th AIAA/ASME /SAE/ASE, Joint Propulsion Conference \& Exhibit, Salt Lake City, Utah, 8-11 July, 2001.

${ }^{11}$ M. Tajmar et al.: Measurement of Gravitomagnetic and Acceleration Fields Around Rotating Superconductors, preprint October 2006.

${ }^{12}$ M. Tajmar et al.: Experimental Detection of the Gravitomagnetic London Moment, http://arxiv.org/abs/gr-qc/0603033, 2006.

${ }^{13}$ M. Tajmar et al.: Measurement of Gravitomagnetic and Acceleration Fields Around Rotating Superconductors, STAIF AIP, February 2007.

${ }^{14}$ Tajmar, M.: http://arxiv.org/abs/0707.3806v5, 14 September 2007, 14 pp. Note: This paper contains a comparison with the measurements by R.D. Graham et al. and also with the Stanford-NASA Gravity Probe B experiment. 
${ }^{15}$ Tajmar, M., Plesescu, F., Seifert, B., Schnitzer, R., and Vasiljevich, I.: Investigation of Frame-Dragging-Like Signals from Spinning Superconductors using Laser Gyroscopes, AIP Conference Proceedings, Vol. 969, 2008, pp. 1080-1090.

${ }^{16}$ M. Tajmar et al:: Anomalous Fiber Optic Gyroscope Signals Observed above Spinning Rings at Low Temperature, arXiv:0806.2271, preprint accepted for presentation at Low Temperature Physics Conference (to be published in Journal of Physics Conf. Proc.), August 2008, 8 pp.

${ }^{17}$ R. D. Graham et al.: Experiment to Detect Frame Dragging in a Lead Superconductor, www2.phys.canterbury.ac.nz/ physrin/papers/SuperFrameDragging2007.pdf), 6 July 2007, 11 pp.

${ }^{18}$ Kiefer, C.: Quantum Gravity, Oxford University Press, 2007.

${ }^{19}$ Ciufolini, I et al.: Determination of frame-dragging using earth gravity models from CHAMP and GRACE, New Astronomy 11 (2006) 527-550.

${ }^{20}$ Veltmann, C.: Facts and Mysteries in Elelementary Particle Physics, World Scientific, 2003.

${ }^{21}$ Zwiebach, R.: Introduction to String Theory, Cambridge Univ. Press, 2004.

${ }^{22}$ Dröscher,W., J. Hauser: Guidelines For a Space Propulsion Device Based on Heim's Quantum Theory, AIAA 2004-3700, 40th AIAA/ASME/SAE/ASE, Joint Propulsion Conference \& Exhibit, Fort Lauderdale, FL, 11-14 July, 2004,31 pp.

${ }^{23}$ Dröscher,W., J. Hauser: Heim Quantum Theory for Space Propulsion Physics, AIP, STAIF, 2005, 10 pp.

${ }^{24}$ Dröscher,W., J. Hauser: Magnet Experiment to Measuring Space Propulsion Heim-Lorentz Force, AIAA 2005-4321, 41st AIAA/ASME/SAE/ASE, Joint Propulsion Conference \& Exhibit, Tuscon, Arizona, 10-13 July, 2005, 10 pp.

${ }^{25}$ Hauser, J., Dröscher,W.: Generation of Gravity-Like Field I: Analysis of Gravitomagnetic Phenomena, to be submitted to arXiv, see www.hpcc-space.de (News) July 2008, 13 pp.

${ }^{26}$ Dröscher,W., J. Hauser: Generation of Gravity-Like Field I: Theoretical Analysis Phenomena, to be submitted to arXiv, see www.hpcc-space.de (News) July 2008, 14 pp.

${ }^{27}$ T. Regitnig-Tillian: Antigravitation: Gibt es sie doch ?, P.M. 3/2007, pp. 38-43 and www.pm-magazin.de/audio.

${ }^{28}$ S. Clark: Gravity's Secret, 11/11 2006, New Scientist, pp.36-39, archive.newscienist.com.

${ }^{29} \mathrm{http}$ ://einstein.stanford.edu/highlights/status1.html: Mission Update: Program Status 23 May 2008.

${ }^{30} \mathrm{H}$. Lietz: 11 Lichtjahre in 80 Tagen. Von der Feldtheorie Burkhard Heims und ihrer Anwendung für einen Raumfahrtantrieb,Telepolis Special 1/2005, Heise Verlag: Hannover, S.47-51.

${ }^{31}$ H. Lietz: Hyperdrive, 7-13 January, 2006, New Scientist.,

${ }^{32}$ H. Lietz: Plus Vite que la Lumière, Scilogic, 2006, pp. 89-94.

${ }^{33}$ Moton-Nkhata,T., Donahue, B., Hauser, J.: Nuclear and Future Flight Propulsion, Aerospace America, December 2007, pp. 60-61. 